\title{
Visual perception of rapidly presented word sequences of varying complexity
}

Four experiments are described that determine whether or not syntactic complexity affects the visual perception of rapidly presented word sequences. The results indicate that sentences containing only one sentence in the underlying structure are more accurately reported than sentences containing two underlying sentences. It is shown that this result is not due solely to distortion of the input, but is likely to reflect the rate at which structural representations of the input can be developed.

The work of Yntema, Wozencraft, and Klem (1964) on short-term memory and Mayzner, Tresselt, and Cohen (1966) on perception of letter sequences suggests that very rapid presentation of successive stimulus elements produces a breakdown in $S$ 's capacity to organize the input sequence. If this interpretation is correct, then similar procedures should have marked effects on the perception of linguistic material. If the number of items correctly reported is a function of the extent to which the input is interpreted within a structural framework, then the perception of word sequences will be more accurate when the words form a sentence rather than a random string. Further, sentences with a complex structure should be more difficult to report than sentences with a simple structure.

Similar perceptual effects have already been found with acoustic presentation under conditions that degrade the sensory input. This is achieved either by presenting the sentence against a background of a loud masking signal (Miller, Heise, \& Lichten, 1951; Miller, 1962; Miller \& Isard, 1963; Mehler \& Carey, 1967) or by filtering out components of the speech signal (Compton, 1967). These signal-degrading techniques have two disadvantages. First, distortion of the input may mean that some information never reaches the sentence processing system at all. Ideally, all the necessary sensory information should be available. Second, if the presentation is auditory, then the time available for sentence analysis to be performed is undetermined. The available evidence suggests that there is an "echoic" storage of acoustic input, and that usable information persists for several seconds after presentation of the stimulus (Neisser, 1968; Crowder \& Morton, 1969). This permits the sentence processor to lag far behind the input.

Visual presentation may provide a technique that avoids these problems. If the individual words of a sentence are consecutively displayed so that each word falls on roughly the same region of the retina, then the occurrence of each new word eliminates the preceding word from the sensory storage system (Kahneman, 1968). Thus, any possibility of a cumulative sensory storage of the input is eliminated, and the $S$ is forced to process each word as it occurs. In this way, it should be possible to push the sentence processing system to the limits of its capabilities. It is also possible that the rate of presentation necessary to accomplish this will be slow enough to avoid the total masking of words, and for each word to be adequately registered in the visual system.

The aim of this paper is to explore the possibility of using rapid serial visual presentation (RSVP) to detect the effects of complexity of sentence structure. The method of presentation of the sentences is similar to that used by Kolers and Katzman (1966) in their study of the perception of rapidly presented letter sequences. Each word was photographed individually on successive frames of a $16-\mathrm{mm}$ film, which was then projected with a variable-speed movie projector. The variable of sentence complexity was manipulated in terms of the number of sentences contained in the deep structure analysis of the input sentence (Chomsky, 1965).

\section{EXPERIMENT 1}

In order to apply a stringent test, it was decided that the sentences to be used should be as simple as possible and well within the immediate memory span. It was also hoped that the criterion of sentence complexity used would be unexceptionable, regardless of the general theoretical position adopted. Sentences were designated as simple if the deep structure contained only one underlying sentence and were designated as complex if the deep structure contained two underlying sentences. It is assumed that the deep structure sentence is a fundamental perceptual unit, and when two such units are involved, it can be argued that the number of possible grammatical relations between constituents is increased (e.g., see Fodor \& Garrett, 1967, fn. 2). In addition, an extra set of relations must be considered in the case of complex sentences, namely, the relations between the sentences themselves. Further, the fact that the analytical routines involved must be called recursively in the case of complex sentences suggests that these sentences may require extra computation time. In this experiment, three types of materials were constructed: simple sentences, complex sentences, and strings of words formed by randomly rearranging the words of simple or complex sentences. While it was expected that these scrambled strings would be reported less accurately than the sentences, as found by Miller and Isard (1963), these strings served a further purpose. Complex sentences differ from simple sentences in various ways, one of them being the number of verbs contained in the sentence. If such a variable by itself produces perceptual effects, then the same effects should be obtained for scrambled strings based on complex or simple sentences.

\section{Method}

Stimulus presentation. Each word to be used in the experiment was printed in black letters on a white background, with the initial letter of each sentence in upper case (lower case for scrambled strings). The individual words were then photographed in the desired sequence using a $16-\mathrm{mm}$ movie camera, with each word occupying one frame. The film was subsequently processed as a negative, which was projected by means of a variable-speed movie projector. Hence, each word was seen as white lettering on a dark background. After considerable pilot work, a projection rate of 16 frames per second was selected. Since each word occupies one frame, with no blank frames in between words, each word is presented for approximately $62.5 \mathrm{msec}$. During this time, the image is twice interrupted very briefly by the shutter of the projector. Thus, the $S$ is required to process the input material at the rate of 16 words a second. Each word was carefully centered in the viewfinder of the camera so that all words would fall on 
approximately the same portion of the retina (provided no eye movements occurred during presentation).

Sentences. All sequences used were six words in length. In deciding whether a sentence contained one or more underlying sentences, reasonably conservative criteria were employed. For example, adjectives were not assumed to involve separate deep structure sentences, nor were auxiliary verbs. This decision was made in spite of linguistic evidence to the contrary, and may well be quite incorrect. The sentences used may be grouped in the following way.

(1) Simple sentences. (a) Predicate constructions (e.g., The young boy was very hungry). (b) Transitives (Alan has broken my mother's vase). (c) Adverbials (The kitten climbed over the fence).

(2) Complex sentences. (a) Reduced relatives (The truck Susan was driving crashed). (b) Unreduced relatives (The clothes that Mary wore vanished). (c) Complements (They persuaded him to work harder). (d) Time adverbials (Bob celebrated after his friends arrived). (e) Manner adverbials (Jim escaped by unlocking the door).

(3) Scrambled strings. (a) Based on simple sentences (box the looked into dog the). (b) Based on complex sentences (sharp they spears equipped advanced with).

There were three items in each of the sentence categories, making 9 simple sentences and 15 complex sentences, and there were five items in each of the scrambled string categories. As indicated before, all the simple sentences were hypothesized to have only one sentence in the deep structure analysis, whereas all the complex sentences involved two underlying sentences. Obviously, the nature of these underlying sentences varied considerably, although none of them took the form of questions, negatives, or passives. In addition, the way in which the underlying sentences of the complex structures are combined to form a surface structure also varies. For example, the complement sentences shared only the feature that some kind of complementation was involved.

Procedure. Prior to the beginning of each sentence or string, two warning signals were presented. The first consisted of the word "ready," which was followed by the signal "XX," which flashed on and off twice, signaling to $S$ that the sentence was about to be presented. The first word was projected approximately $250 \mathrm{msec}$ later. Following the last word, the visual field consisted simply of the dark background seen throughout. In order to prevent $S$ from realizing that all items were of the same length, four dummy items were
Table 1

Mean Number of Words Correctly Reported for Groups of Items

\begin{tabular}{ll}
\hline Simple Sentences & 3.87 \\
Predicates & 3.51 \\
Transitives & 3.71 \\
Adverbial & 4.41 \\
Scrambled Strings & 2.34 \\
"Simple" & 2.26 \\
"Complex" & 2.42 \\
Complex Sentences & 3.51 \\
Reduced Relatives & 3.09 \\
Unreduced Relatives & 3.17 \\
Complements & 4.27 \\
Time Adverbials & 3.63 \\
Manner Adverbials & 3.39 \\
\hline
\end{tabular}

included that contained either three or four words. The order in which the items were presented was determined randomly, but all $S s$ received the same order. Four practice items were included.

The instructions to $\mathrm{S}$ emphasized that he was only to write down what he actually saw. It was explained that he would see three types of items: sentences, scrambled strings of words, and sentences with some of the words missing. It was pointed out that the incomplete sentences were included to discourage guessing. Although no items of this third type were actually included, alk Ss were convinced that a substantial proportion of the sentences were of this form, since they very seldom saw a complete sentence. The Ss were required to write down as many of the words as they could in the order that they were presented, and this was done immediately after each item had been presented. The next item was not presented until each $S$ had completed this task (this required about $10 \mathrm{sec}$ ). The duration of the total experiment was approximately $25 \mathrm{~min}$.

Subjects. Fifty paid volunteer undergraduate students served as Ss. All were naive with respect to the purpose of the experiment. The Ss were run in groups of three to five members.

\section{Results}

The written reproductions were scored in the following way. Each word had to be reported exactly as presented (except for obvious spelling errors), and in the correct position in the sequence. Intrusions of words not presented were ignored. Where order errors occurred, the reproduction was scored so that the number of errors was kept to a minimum (e.g., recalling $A B C D E$ as $C D E A B$ produces only two errors, $A$ and $B$ ).

For each $S$, the mean number of words per sentence correctly reported was determined for each sentence type, and the means are given in Table 1 for all $S s$ combined. Considering first the results for the sentences, analysis of variance of the overall means for complex and simple sentences indicated a significant effect in the predicted direction, $F(1,49)=25.88$, $\mathrm{p}<.001$. Thus the major hypothesis is confirmed: Significantly fewer words are reported when the input sequence must be analyzed into two underlying sentences. Combining the results of the scrambled strings and sentences into a factorial design with two factors, sentences vs scrambled and complex vs simple, it was found that the predicted difference between sentences and scrambled strings was significant, $F(1,49)=277.21, p<.001$. Further, the complexity effects observed for sentences were not observed for the scrambled strings, where the effect was actually completely reversed, and hence there is a marked interaction between the main effects, $F(1,49)=29.78, \quad p<.001$. This result indicates that the complexity effects observed for sentences cannot be attributed to differences in overall distribution of word types, since the scrambled items matched the sentences in this respect.

Inspection of the reproductions revealed that over $80 \%$ of the errors (ignoring the order of the reported items) were omissions. Approximately $10 \%$ of the errors consisted of recognizable distortions of an input item, with occasional intrusions of words totally unrelated to the input. Similar trends were apparent for both the sentences and the scrambled items. However, when the order of report was considered, sentences produced less than $3 \%$ order errors, but for scrambled strings, $14 \%$ of the errors were order errors. However, it is not possible to attribute the poorer performance on scrambled items solely to increased difficulty in arranging the words in the correct order. When these items are rescored without taking order into account, the overall mean is lifted to 2.88 , which still falls far short of the mean for complex sentences. In fact, only 4 of the $50 \mathrm{Ss}$ fail to show better performance on the complex sentences.

From a phenomenological point of view, there seemed to be some consensus that in the vast majority of cases the words that were omitted from the report were simply not seen at all rather than being unclear or illegible. However, it seems likely that phenomenal report is of limited usefulness, since there appear to be cases where the $S$ cannot recall seeing some items but has apparently made use of the information conveyed. For example, during pilot experiments, many Ss reported that they felt they knew what was in the sentence, although they were certain they had not seen some of the words. It was very seldom the case that these words were entirely 
Table 2

Probability of Correct Report of Words of Varying Length

Length in Letters

\begin{tabular}{llllllll} 
& 2 & 3 & 4 & 5 & 6 & 7 & $8+$ \\
\hline Probability of Correct Report & .43 & .44 & .57 & .67 & .74 & .81 & .82 \\
\hline
\end{tabular}

predictable from context (e.g., the word "shabby" in Both priests wore shabby sandals).

The failure to detect some words may be an indication that S's processing system was momentarily overloaded, or it may simply indicate that the physical properties of the word itself, or the adjacent words, were such that accurate identification was impossible. Eriksen (1966) has emphasized that the temporal summation of successively presented signals may effectively degrade the input signal. In this case, it could be argued that each word is effectively seen as being superimposed on the decaying trace of the preceding word. One obvious physical property that should be taken into account is word length. The data presented in Table 2 indicate that in this experiment there was a marked tendency for longer words to be reported more often than shorter words. This may be due to the fact that since relatively long words are more likely to be surrounded by words of shorter length, the beginnings and ends of the longer words would be more likely to be free of interference from surrounding words (all words were centered in the middle of the screen). For any particular word, the probability of detection was .59 if the preceding word was shorter in length, but only .49 if the preceding word was longer. Similarly, if the word following was shorter, the probability of detection was .58 as opposed to .51 if the word following was longer. However, it is fortunately the case that the average word length for the simple sentences (4.46 letters) was less than for the complex sentences (4.66 letters), and hence the factor of word length could only have gone against the hypothesis. A further possibility is that the pattern of alternation between long and short words may influence detectability, and that this variable was confounded with the complexity variable. In order to test this notion, the sum of the absolute differences in the lengths of adjacent words was computed for each sentence or string. A low value means that adjacent words tended to be of similar lengths. While the simple sentences tended to produce higher values of this statistic (11.8 as opposed to 10.9 for complex sentences), the same trend was true for the scrambled strings based on simple or complex sentences (11.8 as against 8.0). Since the simple-complex effects for scrambled strings were the reverse of those for the sentences, it would be difficult to explain the results on the basis of word-length alternation. Further, classification of each sentence according to its alternation pattern failed to exert a significant effect on the mean number of words reported, $F(2,24)=0.56, p>.05$.

In Fig. 1 the probability of long and short words being reported is plotted as a function of serial position in the sentence. The shape of these curves is reminiscent of the serial position effects for long- and short-term memory, but the connection may be only accidental. Since the last word is followed only by a relatively dark background, it is effectively presented for a longer period than any other word. That is, no backward masking effects occur. Similarly, since no word precedes the first, forward masking effects will be absent for this word. Thus, on purely sensory grounds, the first and last words should be better reported than others. However, this is not necessarily so for the comparison between, say, the third and fifth words.

Finally, there were no strong practice effects. Considering the results for sentences, the mean number of words per sentence reported for successive blocks of nine sentences each were $3.93,3.43$, and 3.66 .

\section{Discussion}

In general, the results strongly confirm the hypothesis that the number of words reported is a function of the linguistic organization that can be imposed on the presented sequence. Performance is worst for scrambled strings where no such organization is possible. Further, there are between-sequence effects that correspond to the complexity of the structure that must be assigned. However, the results also indicate that the probability of detection of a word is affected by variables completely unrelated to structural processing. The existence of a strong word-length effect points to the operation of purely visual processes and suggests that some words may never be adequately registered in the visual system. Although it is not possible to argue that variations in word length could account for the complexity effects, future experiments should be designed with better control of this variable. In addition, there is a clear effect of serial position. Both effects make it extremely difficult to draw conclusions about the perceptibility of individual words.

These considerations also raise difficulties for the comparison of sentences with different syntactic structure. To some extent, variations in syntactic structure will always be confounded with serial position effects. For example, comparison of That he came surprised us with It surprised us that he came is complicated by the difference in serial position of critical items, such as the verb surprised. Any difference between these sentences may be due solely to this fact. For this reason, it is not possible to draw conclusions about between-sentence effects based on data from a small number of sentences of similar structure. However, there are trends apparent in the data that merit further examination. For example, the means reported for the complement sentences in Table 1 are far higher than their classification as complex sentences would suggest. Also, there is no marked difference between the reduced and unreduced relatives (in the reduced relative, the relative pronoun is deleted), and these constructions have already been shown to differ in complexity (Fodor \& Garrett, 1967; Foss \& Lynch, 1969; Hakes \& Cairns, 1969). Whether these problems indicate a deficiency in theory or technique remains to be settled.

The most interesting aspect of the experiment is the problem of determining what happened to the words that were not reported. There appear to be three possibilities. First, both forward and backward masking may serve to degrade the input, since each successive word is

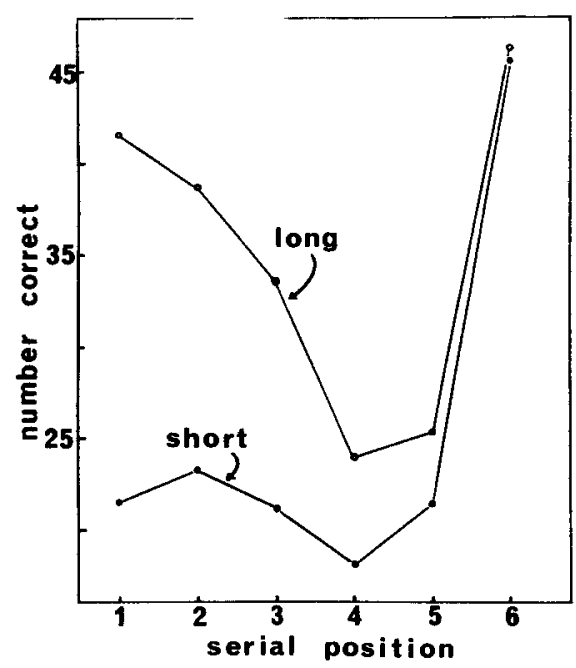

Fig. 1. Mean number of Ss correctly reporting long words (five or more letters) and short words (less than five letters) as a function of serial position in the sentence. 
effectively projected onto the decaying trace of the preceding word. Whether temporal summation, erasure, or metacontrast is involved, it could be argued that the unreported words were never presented clearly enough to be identified. The fact that more words are unreported in the case of the complex sentences or the scrambled items may simply indicate that $S$ used contextual information to reconstruct the missing parts of the sentence, and that this procedure is more efficient in the case of simple sentences. ${ }^{2}$ The strongest evidence for this view is the reported relationship between detection and word length. Second, there is an alternative view of temporal masking (e.g., Haber \& Standing, 1968), in which the masking stimulus is seen as affecting the time available for processing, without affecting the adequacy of registration of the input. Thus, each word may have been potentially identifiable, but under pressure, $S$ may not have attended fully to each word before the next word was presented. The third view involves similar assumptions, but emphasizes the rate at which information can be transferred from visual storage to short-term memory (Sperling, 1967; Mewhort, Merikle, \& Bryden, 1969). On this view, the unreported items may be attended to, but may not be transferred to STM rapidly enough, and hence are forgotten. Yntema, Wozencraft, and Klem (1964) obtained such an effect with rapid acoustic presentation of time-compressed digits that were reported to be clearly identifiable. On this interpretation, the S's primary task is that of assimilating each new word as rapidly as possible to a structural representation of the sentence held in STM. If the structural representation proves to be incorrect, insufficiently developed, or requires substantial modification to accommodate further input (e.g., the addition of a new deep structure sentence), then the assimilation of new words to the structure may be momentarily delayed while the structure is appropriately modified. But this will produce a bottleneck in the transfer operation, with the result that some words will never be transferred to STM. The existence of a serial position effect is suggestive of such a process. In the remainder of the report, three experiments are reported that were designed to yield information relevant to these various interpretations.

\section{EXPERIMENT 2}

If the first alternative is correct, then the unreported words are never effectively registered in the visual system. If the third view is correct, then the unreported words are never successfully transferred to STM.
In this experiment, an attempt was made to eliminate the possibility that any item could be forgotten. The Ss viewed sentences and scrambled strings under two conditions. In the first condition, Ss were given the initial pair of letters of one of the words in the input sequence, and were asked to identify this word. This condition is referred to as the "monitor" condition. In the second condition, Ss attempted to recall the entire input sequence in the usual manner. A comparison was then made of the probability of detection of a particular word under the monitor conditions and the probability of recall of the same word when $S$ was attempting to recall the entire sequence. If all words are potentially identifiable, then the monitor condition should produce perfect performance. If some words are forgotten, then performance in the monitor task should be substantially better than in the recall task.

\section{Method}

Procedure. Twelve sentences, each of six words, were prepared. Each sentence contained one critical word, termed the "target" word, that was five, six, or seven letters in length and that was in the second, third, fourth, or fifth serial position. Each sentence was presented under four conditjons: either as a sentence or in a scrambled order (but with the target word left in its original position); for each of these conditions, Ss were instructed either to monitor the sequence for a word of specified length beginning with the pair of letters nominated by $E$ just prior to presentation or to recall the entire sequence. For each of these four presentations of a given sentence, 10 different $S$ s were run. Each $S$ received a block of items for monitoring, followed by a block of items for recall, or vice versa. The order in which the items were presented was such that serial effects were distributed equally over the four conditions. The presentation rate was 12 words per second. The preparation of the film sequence was the same as in Experiment 1, except that only one warning signal, the word "ready," was presented $250 \mathrm{msec}$ before the sequence began. The instructions to $S$ explained that both sentences and random strings of words would be presented, and it was emphasized that Ss should only report what they had actually seen. Four practice items were included.

Subjects. Forty paid voiunteer undergraduates served as Ss, and they were run in small groups of two to five members.

\section{Results}

The main results of interest are
Table 3

Mean Number of Ss Reporting Words as a Function of Task Instructions and Length of Target Word (Maximum $=10$ )

\begin{tabular}{lccc} 
& \multicolumn{3}{c}{ Number of Letters in Target Word } \\
\cline { 2 - 4 } & 5 & 6 & 7 \\
\hline Monitor & 5.75 & 7.63 & 8.75 \\
Recall & 3.25 & 6.13 & 8.00 \\
\hline
\end{tabular}

presented in Table 3, which gives the number of Ss reporting target words of varying lengths under the monitor or recall instructions. It is clear that performance on the monitor task falls short of the maximum possible, but it seems equally clear that the recall performance does not match the monitor performance. Analysis of variance of these means ( 3 by 2 by 4 by 2 factorial using as error term the pooled higher-order interactions) revealed that the effect of word length was significant, $F(2,23)=27.44, p<.001$, and also that the monitor-recall difference was significant, $F(1,23)=13.52, \quad p<.001$. However, no other effects were significant. Thus, no significant serial position effect was observed (there was no effect at all for the monitor task, with some trend for the recall task, but the interaction of task and serial position was not significant, $F(3,23)=2.96, p>.05)$, nor was there any significant difference between sentences and scrambled sequences. The absence of strong serial position effects can be explained readily enough, since the initial and final positions were not considered in this analysis (examination of the recall data for the sentences alone revealed a strong position effect similar to that reported for Experiment 1). However, there is no obvious explanation for the absence of any difference between sentences and scrambled items. The same result was obtained when the recall data were rescored in terms of the total number of words reported (ignoring order errors). A mean of 3.73 was obtained for the sentences, as opposed to a mean of 3.74 for scrambled items.

It appears likely that many of the variables influencing performance on the monitor task also influence performance on the recall task. The product-moment correlation between the number of $\mathrm{Ss}$ successfully monitoring each item and the number of $S s$ recalling the same items was $.70(p<.01)$. Inspection of the scatterplots revealed no evidence of a curvilinear relationship.

Examination of the reproductions of the scrambled items revealed a tendency for Ss to rearrange the words to form a grammatical sequence. The same tendency had been noted in the first experiment, where, for example, the sequence John 
Table 4

Mean Proportion of Grammatical Adjacencies in the Report of Scrambled Items as a Function of the Number of Words Reported

\begin{tabular}{lllllll}
\hline & \multicolumn{4}{c}{ Number of Words Reported } \\
\cline { 2 - 6 } & 2 & 3 & & 4 & 5 & 6 \\
\hline $\begin{array}{l}\text { Proportion of } \\
\text { Grammatical } \\
\text { Adjacencies }\end{array}$ & .03 & .14 & .33 & .54 & .75 \\
\hline
\end{tabular}

father's was nearly always reported as John's father. In some extreme cases, the entire sequence was reported as a fully grammatical sentence. Further, there appeared to be a tendency for this grammatical rearrangement to occur more often when a relatively large number of words had been detected. In order to check on this possibility, a count was made of the number of adjacent words in each reproduction that were also adjacent in one of the possible grammatical rearrangements of the original scrambled sequence. This was expressed as a proportion of the total possible number of such adjacencies (equal to $\mathrm{N}-1$, where $\mathrm{N}$ is the number of words reported). Thus a score of 1.00 means that all words reported formed a grammatical sequence, and a score of 0.00 means that none of them formed a grammatical sequence. In those cases where Ss correctly reported adjacent words that had already constituted a grammatical sequence in the original input, the data were omitted from the analysis. Further, if a grammatical sequence was produced without $S$ making an order error (e.g., reporting only the words we laughed from the input sequence boys we when jumped the laughed), the adjacent pair was treated as being ungrammatical. Thus we are only considering cases where $S$ rearranges the order of the input items. In Table 4 the means of the proportions of grammatical adjacencies are given for varying numbers of words reported. Analysis of variance of these means revealed a significant effect of the number of words reported, $F(4,229)=21.09, p<.001$. As expected, the tendency to rearrange the input in a grammatical order increases steadily as the number of words reported increases.

\section{Discussion}

As might have been expected, the results of this experiment do not unequivocally support the view that the unreported words are never effectively registered, or the view that these words are never assimilated to STM. The latter view is supported by the fact that performance on the monitor task is superior to performance on the recall task. However, the importance of inadequate registration is indicated by the fact that performance on the monitor task falls short of the maximum possible, and also by the fact that both kinds of task are affected by the variable of word length (there is no obvious connection between word length and the time required to transfer words to STM, which will explain why longer words are more easily transferred). Thus the appropriate conclusion appears to be that words may be unreported either because of inadequate registration in the visual system, or because they are lost during transfer to STM.

From the standpoint of a theory of sentence perception, the fact that $S$ s tend to reorganize the scrambled strings to form grammatical sequences is of considerable interest. It is possible that this is not a perceptual effect, and that the reordering takes place only at the output phase. That is, $S$ receives and stores items in the presented order, but rearranges them prior to output. However, this seems unlikely The Ss were instructed to report the items in the order presented, and hence there would be little point in changing the order. Further, it is no simple matter to perform the operations required. With acoustic presentation of six-word scrambled strings, several seconds appear to be necessary to find a grammatical reordering, during which time $S$ must rehearse the input string. If this is so, then there seems to be no reason why $S$ should not report the perceived input order immediately.

A more likely interpretation is that $S$ perceives the words to be in the order he reports them. More precisely, the relative positions occupied by words in STM do not correspond perfectly to the order in which they were transferred from the visual system. Such a result is not surprising if one considers the fact that temporal resolving power must be limited at very fast presentation rates. Further, if $S$ is seen as building up a possible structura representation of the input, then it is not surprising that the order of the input is not always preserved. Words may be assigned to positions within a structure on the basis of their possible syntactic role, and hence their reported order will be dictated by the structure. But the important point is that the extent to which this happens is so closely associated with the number of words reported. This finding presents the strongest evidence against the argument that the number of words reported is a function of purely visual parameters alone. In this case, the input sequence is constant for all Ss, and hence the visual signal is constant. But if $S$ interprets the input in sentential form, he is able to report many more words. This seems to be clear evidence that performance in the recal task is strongly influenced by factors operating after the word has been transferred from the visual system.

If this process always occurred, there would be no reason to expect scrambled sequences to be perceived less readily than sentences. This may explain the absence of any difference between these types of items in this experiment. In Experiment 1, a faster presentation rate was used (16 words per second as opposed to 12), and this may have prevented $S$ from reorganizing the material.

\section{EXPERIMENT 3}

It is possible that many of the effects reported so far are solely a function of the information value of the messages. The $S$ may be able to exploit the redundancy of English, and reconstruct the items that were not clearly received. Clearly, this would be difficult to do in the case of scrambled items, and it may be that it is also more difficult to reconstruct complex sentences than simple sentences. The present experiment was designed to test this hypothesis. Blurred typewritten copies of simple sentences, complex sentences, and scrambled sequences were given to $\mathrm{Ss}$ who were asked to write down as much as they could, with no time limit. If the redundancy of complex sentences is lower than that of simple sentences, then fewer words should be reported for the complex sentences. Similar effects should be observed for the comparison of sentences and scrambled sequences.

\section{Method}

A sample of six complex and six simple sentences was constructed. All sentences were six words in length. The simple sentences were: (1) Someone left the ugly vase inside. (2) The rabbit jumped into the truck. (3) Betty will send Mary the letter. (4) The dogs played with the girls. (5) Susan emptied the box of cigars. (6) The boys have seen several lions. The complex sentences were: (1) Knowing Jim made Betty very happy. (2) The kitten nobody liked was young. (3) Pretty girls that object mav leave. (4) You think nothing made it start. (5) Alan wounded the man we stopped. (6) Why John liked going nobody knew. It will be noticed that each simple sentence is matched with a complex sentence, such that the length of each word in the simple sentence was always within one letter (plus or minus) of the length of the word in the complex sentence in the corresponding ordinal position.

As in Experiment 1, complexity is determined by the number of sentences in the deep structure analysis, all simple sentences having one (ignoring adjectives), and all complex sentences having two, except for the fourth, which has three. In addition to the above items, a sample of 12 scrambled sequences was constructed, half of them by scrambling a simple sentence 
Table 5

Mean Number of Words Correct Per Item for Visually Degraded Sequences of Varying Complexity

\begin{tabular}{lcc}
\hline & Simple & Complex \\
\hline Sentences & 3.82 & 3.75 \\
Scrambled & 2.29 & 2.14 \\
\hline
\end{tabular}

(not one of those above), half by scrambling a complex sentence. All items were arranged in a random order and were embedded in a series of 16 other items. These 40 items were then typed, one item per line, such that the impressions produced were extremely blurred. This was achieved by taking the 13th carbon copy typed on an electric typewriter. The initial letter of each sentence was in upper case, but was in lower case for the scrambled items. No punctuation was included. It was explained to the Ss that some of the items were sentences and some were random strings of words, and that they had ample time in which to write down everything they could. Twelve paid volunteer undergraduates served as Ss, and were tested in a single session. All Ss completed the 40 items within half an hour.

\section{Results and Discussion}

The mean number of words correctly reported for each condition is given in Table 5. The difference between simple and complex sentences is extremely small and is not statistically significant $t(11)=0.43, p>.05$. Similarly, the effect of complexity on scrambled items was not significant, $\mathrm{t}(11)=1.48, \mathrm{p}>.05$. It could be argued that little has been demonstrated by this failure to reject the null hypothesis. This may indicate only that the experiment was insensitive, either because of the small number of Ss involved or because of error variance. However, the experiment was sufficiently sensitive to reveal a strong difference between sentences and scrambled items, $\mathbf{t}(11)=9.48, \mathrm{p}<.001$. There seems to be no obvious reason why the experiment should fail to detect the effects of redundancy in one case but not in the other. Thus it is concluded that visual degrading per se has no differential effect on sentences with varying numbers of deep structure sentences, but does have marked differential effects on sentences and nonsentences. From this it is inferred that the better performance on simple sentences reported in Experiment 1 cannot be due to the presumed variation in information value of complex and simple sentences. However, the reported differences between sentences and scrambled items could well be due to the increased information value of the scrambled items.

\section{EXPERIMENT 4}

It has been suggested that the effects of complexity on performance in the RSVP situation are a direct consequence of the rate of presentation. The purpose of this experiment was to examine whether decreasing the rate of presentation would diminish the effects of complexity. This presents certain design problems, since a substantial decrease in rate of presentation leads to vastly improved performance, so that errors become very scarce and strong ceiling effects are encountered. These problems were overcome by placing a masking stimulus between each of the words in the sentence. This has the effect of decreasing the number of words reported and balances the effects of decreasing presentation rate. Thus the overall difficulty of the task does not change drastically. But in the case of the slower presentation rate, there is considerable "dead" time in between words (while the mask is being presented), in which there should be ample time to perform the necessary syntactic operations and the assimilation of items to STM. Thus it would be expected that the number of deep structure sentences would exert little influence on the number of words reported.

\section{Method}

Sentences. The sentences used in this experiment were the sample of simple and complex sentences used in the previous experiment. These have been shown to be of equal information value, and the fact that sentences were matched in terms of word lengths is an obvious advantage in a situation where word length is a relevant variable.

Presentation of sentences. Two conditions of presentation were used. In the "no mask" condition, the sentences were filmed in the way described in Experiment 1, that is, each word occupying a single frame. These sentences were presented to Ss at 16 words per second. In the "mask" condition, a masking stimulus appeared on the frame preceding each word and on the frame succeeding each word. The masking stimulus consisted of a left-to-right array of either four or nine letters centered in the screen, with the letters appearing in various orientations, some inverted, some rotated through $120 \mathrm{deg}$, etc. These sentences were projected at eight frames per second, which yields a presentation rate of four words per second. 3

Procedure. The items involved in this experiment were presented to $S s$ at the conclusion of the item presentation in Experiment 2. In this segment of the testing session, all Ss first received three simple and three complex sentences in alternating sequence presented under the "no mask" conditions. The nature of the masking stimulus was explained, and Ss then received practice items. Then followed a sequence of three complex and three simple sentences in alternating order, presented under the "mask" conditions. The Ss were told to ignore the mask and write down the words in the order that they were presented. Two films were constructed, and the sentences assigned to the mask condition on one film were assigned to the no-mask condition on the other. Approximately half of the Ss saw each film.

Subjects. The Ss for this experiment were the same as those used in Experiment 2. The data from one $S$ were lost, leaving an $\mathrm{N}$ of 39 .

\section{Results and Discussion}

The mean number of words reported per sentence for the two presentation conditions is plotted in Fig. 2. Analysis of variance of these means revealed a significant effect of complexity, $F(1,38)=39.67, p<.001$, confirming the results of Experiment 1. The effects of the presentation conditions were also significant, $\quad F(1,38)=12.27, \quad p<.01$, indicating that overall, performance was better under the "mask" condition (remember that this condition involves a very slow word presentation rate).

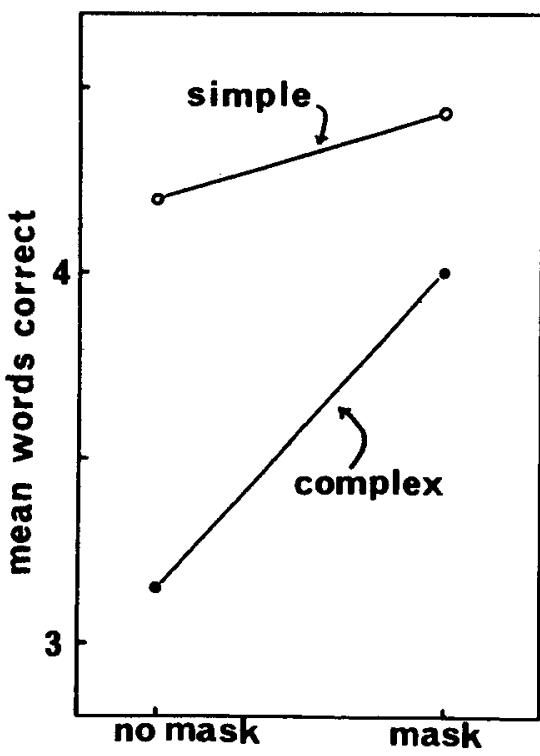

Fig. 2. Mean number of words reported for complex and simple sentences under the "no mask" condition (16 words per second) and "mask" condition (4 words per second). 
However, performance was well below the maximum possible (six words correct per sentence), so that ceiling effects are not involved. As indicated in Fig. 2, there is a marked interaction between the effects of complexity and the presentation conditions, and this effect was significant, $F(1,38)=15.27, p<.001$. Thus the major hypothesis is confirmed; there is a significant reduction in the effects of complexity for the slower presentation rate. However, it is of interest to note that the effects of complexity are by no means eliminated entirely

It may be argued that the observed interaction is a result of the fact that the masked sentences were always presented after the "no mask" sentences when Ss have gained more practice at the task. However, all Ss had just previously viewed 24 sentences as part of a previous experiment, and hence were reasonably well practiced (in any case, there are no indications of strong practice effects) Alternatively, it could be argued that the masking distracts $S$ from the task at hand and prevents him from performing sentential analysis at peak efficiency Although this might be expected to lead to a decrease in the difference between complex and simple sentences (it seems more likely to have the reverse effect), the degree of distraction involved cannot be very great, since performance improved under the masking conditions.

\section{GENERAL DISCUSSION}

The major aim of this paper has been to discover if the perceptual complexity of sentences can be revealed by a technique that neither degrades the input nor relies on the use of highly elaborate and complex sentences. The evidence produced in Experiment 1 strongly suggests that the rapid visual exposure of successive elements of the sentence produces perceptual effects that appear to be related to the structure of the input sentence. However, the strong word-length effect observed in this experiment suggests that word detection is also a function of purely visual parameters. Three sources of evidence indicate that this variable was not responsible for the complexity effects. First, the average word length of simple sentences was shorter than for complex sentences. Second, in Experiment 4, complex and simple sentences were matched for word length, and strong complexity effects were still obtained. Third, in Experiment 2 the accuracy of perception of scrambled sequences was shown to be a function of the extent to which the input was reorganized into sentential form. In this case, accuracy cannot be a function of visual parameters, since the input is held constant.

However, it has not been established that the RSVP technique does not degrade the stimulus material. In Experiment 2 , where Ss were required only to monitor for a single target word, performance fell short of the maximum possible. Further, detection of the target word was also a function of word length. However, it was also demonstrated that performance on the monitor task was superior to recall; this indicates that at least some of the words that are not recalled were clearly registered in the visual system. This suggests that some words are never effectively transferred to STM.

Finally, it is concluded that complexity of sentence structure only affects perception when the time available for sentence analysis is severely limited. In Experiment 4, the presentation rate was substantially decreased without markedly affecting the overall difficulty of the task. Under these conditions, the complexity effects were diminished, but were not entirely eliminated. ${ }^{4}$ In addition, the blurred visual presentation of sentences used in Experiment 3 failed to show differences between complex and simple sentences. In this situation, there is no limitation imposed on sentence processing time, and the only variable affecting performance is the degree of distortion of the input. Thus it is inferred that distortion alone does not produce perceptual effects related to structural complexity.

\section{REFERENCES}

CHOMSKY, N. Aspects of the theory of syntax. Cambridge, Mass. M.I.T. Press, 1965.

COMPTON, A. J. Aural perception of sentences of different syntactic structures and lengths. Language \& Speech, 1967, 10, 81-87.

CROWDFR, R. G., \& MORTON, J Precategorical acoustic storage (PAS) Perception \& Psychophysics, 1969, 5 365-373.

FRIKSEN, C. W. Temporal luminance summation effects in backward and forward masking. Perception \& Psychophysics, 1966, 1 , 87-92.

FODOR, J. A., \& GARRETT, M. Some syntactic determinants of sentential complexity. Perception \& Psychophysics, 1967, 2, 289-296.

FOSS, D. J. \& LYNCH, R. H. Decision processe during sentence comprehension: Effects of surface structure on decision times. Perception \& Psychophysics, 1969, 5, 145-148.

HABER, R. N., \& STANDING, L. Clarity and recognition of masked and degraded stimuli. Psychonomic Science, 1968, 13, 83-84

HAKES, D. T., \& CAIRNS, H. S. Do relative pronouns affect sentence comprehension? raper presented at the meetings of the
Midwestern Psychological Association, Chicago, 1969.

KAHNEMAN, D. Method, findings, and theory in studies of visual masking. Psychological Bulletin, 1968, 70, 404-425.

KOLERS, P. A., \& KATZMAN, M. T. Naming sequentially presented letters and words. Language \& Speeth, 1966, 9, 84-95.

MAYZNER, M. S., TRESSELT, M. E., \& COHEN, A. Preliminary findings on some effects of very fast sequential input rates on perception. Psychonomic Science, 1966, 6, 513-514.

MEHLER, J., \& CAREY, P. The role of surface and base structure in the perception of sentences. Journal of Verbal Learning \& Verbal Behavior, 1967, 6, 335-38.

MEWHORT, D. J. K., MERIKLE, P. M., \& BRYDEN, M. P. On the transfer from iconic to short-term memory. Journal of Experimental Psychology, 1969, 81, 89-94.

MILLER, G. A. Decision units in the perception of speech. IRE Transactions on Information Theory, 1962, IT-8, 81-83.

MILLER, G. A., HFISE, G. A., \& LICHTEN, W. The intelligibility of speech as a function of the context of the test materials. Joumal of Fxperimental Psychology, 1951, 41, 329-335.

MILLER, G. A., \& ISARD, S. Some perceptual consequences of linguistic rules. Journal of Verbal Learning \& Verbal Behavior, 1963, 2, 217-28.

NEISSER, U. Cognitive psychology. New York: Appleton-Century-Crofts, 1967.

SPERLING, G. Approximations to a model of short-term memory. Acta Psychologica, 1967, $27,285-292$.

YNTEMA, D. B., WOZENCRAFT, F. T., \& KLEM, L. Immediate serial recall of digits presented at very high rates. Paper presented to Psychonomic Society, 1964.

\section{NOTES}

1. Address: Department of Psychology, Monash University, Clayton, Victoria, Australia 3168.

2. It is our conviction that Ss very seldom attempted to guess the words that were not seen. They were instructed not to guess, and were led to believe that many sentences were presented in an incomplete form. In a substantial proportion of incomplete reports, the missing words were highly predictable (e.g., John wanted me - come home), yet Ss still refrained from guessing.

3. It was originally intended to project these sentences at 16 frames per second, producing a constant exposure duration of words in both the "mask" and "no mask" conditions. However, at this presentation rate, none of the pilot Ss ever reported a single word. The confounding of the presentation rate with exposure duration is not considered to be important. The design merely requires a slower presentation rate under conditions that preserve roughly the same error rate as that obtained for the faster rate.

4. The presence of the masking stimulus may still serve to limit the time available for sentence analysis. With the offset of each word, S must still spend time analyzing the mask to determine whether this is a word or not. Apparently, a mask consisting of disoriented letters is far more disruptive than a mask consisting of a word (see Note 3 ).

(Accepted for publication December 11, 1969.) 